MATHEMATICS OF COMPUTATION

Volume 80, Number 274, April 2011, Pages 1197-1219

S $0025-5718(2010) 02420-2$

Article electronically published on September 13, 2010

\title{
THE NUMBER OF LATIN SQUARES OF ORDER 11
}

\author{
ALEXANDER HULPKE, PETTERI KASKI, AND PATRIC R. J. ÖSTERGÅRD
}

\begin{abstract}
Constructive and nonconstructive techniques are employed to enumerate Latin squares and related objects. It is established that there are (i) 2036029552582883134196099 main classes of Latin squares of order 11; (ii) 6108088657705958932053657 isomorphism classes of one-factorizations of

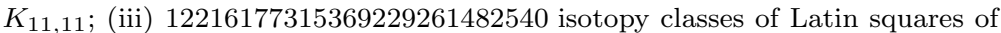
order 11; (iv) 1478157455158044452849321016 isomorphism classes of loops of order 11; and (v) 19464657391668924966791023043937578299025 isomorphism classes of quasigroups of order 11. The enumeration is constructive for the 1151666641 main classes with an autoparatopy group of order at least 3.
\end{abstract}

\section{INTRODUCTION}

1.1. Latin squares. A Latin square of order $n$ is an $n \times n$ array over $n$ symbols such that every row and column is a permutation of the symbols. Latin squares are among the most fundamental combinatorial objects, and have many equivalent formulations [1, Theorem 1.11].

Latin squares are easily seen to exist for every $n$. It is, however, considerably more challenging to count the number of distinct Latin squares. In fact, the exact number is known only for $n \leq 11$, with the most recent case $n=11$ settled by McKay and Wanless [14.

Here our interest is to enumerate Latin squares up to equivalence, whereby we focus on main classes (see \$1.3). We refer to 13 for a brief historical account of enumerating small $(n \leq 6)$ Latin squares; this history goes as far back as the eighteenth century and includes pioneering combinatorialists such as Cayley and Euler. There is a unique main class for $n \leq 3$, two main classes for $n=4,5$, and 12 main classes for $n=6$. There are 147 main classes for $n=7$; this classification was carried out by Norton [15] and corrected by Sade [17]. Kolesova, Lam, and Thiel [9] showed that there are 283657 main classes for $n=8$. All of these results were constructive, that is, representative Latin squares were (or could have been) obtained from each main class. Recently, McKay, Meynert, and Myrvold [13], on the other hand, counted in a nonconstructive way the number of main classes for $n=9$ and $n=10$; these numbers are 19270853541 and 34817397894749939 , respectively.

In this paper, we enumerate the main classes for $n=11$ together with related objects such as quasigroups and loops (see [13] and \$5]).

Received by the editor September 18, 2009 and, in revised form, February 4, 2010.

2010 Mathematics Subject Classification. Primary 05B15, 05A15, 05C30, 05 C70.

Key words and phrases. Enumeration, Latin square, main class.

(C)2010 American Mathematical Society Reverts to public domain 28 years from publication 
Theorem 1. There are

(i) 2036029552582883134196099 main classes of Latin squares of order 11;

(ii) 6108088657705958932053657 isomorphism classes of one-factorizations of $K_{11,11}$;

(iii) 12216177315369229261482540 isotopy classes of Latin squares of order 11;

(iv) 1478157455158044452849321016 isomorphism classes of loops of order 11; and

(v) 19464657391668924966791023043937578299025 isomorphism classes of quasigroups of order 11.

1.2. Group actions. We assume familiarity with group actions [16. The OrbitStabilizer Theorem provides the methodological foundation for counting in [13] as well as in this paper; a similar approach is also used in [7] in the context of onefactorizations of a complete graph.

Let $\Gamma$ be a finite group that acts on a finite set $\mathscr{L}$. For an $X \in \mathscr{L}$, denote the orbit of $X$ by $[X]=\{\gamma X: \gamma \in \Gamma\}$ and the stabilizer of $X$ in $\Gamma$ by $\Gamma_{X}=\{\gamma \in \Gamma$ : $\gamma X=X\}$. For a subgroup $\Pi \leq \Gamma$, denote the conjugacy class of $\Pi$ in $\Gamma$ by $[\Pi]=$ $\left\{\gamma \Pi \gamma^{-1}: \gamma \in \Gamma\right\}$ and the normalizer of $\Pi$ in $\Gamma$ by $\Gamma_{\Pi}=\left\{\gamma \in \Gamma: \gamma \Pi \gamma^{-1}=\Pi\right\}$. The Orbit-Stabilizer Theorem connects the size of an orbit with the size of a stabilizer subgroup by $|\Gamma|=|[X]| \cdot\left|\Gamma_{X}\right|$.

In the context of combinatorial enumeration, the elements of $\mathscr{L}$ are called "labeled" objects, and the orbits of $\Gamma$ on $\mathscr{L}$ are called "unlabeled" objects. Our interest is to count the unlabeled objects. To this end, denote by $N_{i}$ the number of orbits $[X]$ with $\left|\Gamma_{X}\right|=i$. Because $\mathscr{L}$ partitions into orbits, we have

$$
|\mathscr{L}|=|\Gamma| \sum_{i} \frac{N_{i}}{i}
$$

If we now know $|\Gamma|,|\mathscr{L}|$, and $N_{i}$ for each $i \geq 2$, then we can solve (11) for $N_{1}$ and obtain the number of unlabeled objects $\sum_{i} N_{i}$. In the earlier studies [7, 13, the approach is to determine $|\mathscr{L}|$ by a nonconstructive counting technique and the values $N_{i}$ for $i \geq 2$ by a constructive classification up to isomorphism.

In this paper, we extend the nonconstructive tools to the setting of prescribed symmetry, which enables us to determine the values $N_{i}$ nonconstructively also for $i>1$. There are two high-level ideas. First, the stabilizer subgroups of the elements of an orbit form a conjugacy class of subgroups of $\Gamma$. Thus, we can split the task of determining $N_{i}$ into subtasks indexed by conjugacy classes $[\Pi]$ with $|\Pi|=i$. Second, we can solve each such subtask via an analog of (11). Namely, instead of counting individual labeled objects $X \in \mathscr{L}$ as in (1), we count formal pairs $(X, \Sigma)$ consisting of a labeled object $X \in \mathscr{L}$ and a subgroup $\Sigma \leq \Gamma_{X}$ with $\Sigma \in[\Pi]$.

Let us now proceed with a more detailed treatment. Denote by $N_{[\Pi]}$ the number of orbits $[X]$ with $\Gamma_{X} \in[\Pi]$. Taking the sum over all conjugacy classes $[\Pi]$ with $|\Pi|=i$, we have

$$
N_{i}=\sum_{[\Pi]:|\Pi|=i} N_{[\Pi]} .
$$

For a conjugacy class $[\Pi]$, define the following two sets of formal pairs:

$$
\begin{aligned}
& \mathscr{L}_{[\Pi]}=\left\{(X, \Sigma): X \in \mathscr{L}, \Sigma \leq \Gamma_{X}, \Sigma \in[\Pi]\right\}, \\
& \mathscr{C}_{[\Pi]}=\left\{(X, \Sigma): X \in \mathscr{L}, \Sigma<\Gamma_{X}, \Sigma \in[\Pi]\right\} .
\end{aligned}
$$


For $X \in \mathscr{L}$, let

$$
S_{[\Pi]}(X)=\left\{\Sigma \leq \Gamma_{X}: \Sigma \in[\Pi]\right\} .
$$

Observe that $S_{[\Pi]}(\gamma X)=\gamma S_{[\Pi]}(X) \gamma^{-1}$ for all $\gamma \in \Gamma$. By the Orbit-Stabilizer Theorem, we thus have

$$
\left|\mathscr{L}_{[\Pi]}\right|=|\Gamma| \sum_{[X]} \frac{\left|S_{[\Pi]}(X)\right|}{\left|\Gamma_{X}\right|}, \quad\left|\mathscr{C}_{[\Pi]}\right|=|\Gamma| \sum_{[X]:|\Pi|<\left|\Gamma_{X}\right|} \frac{\left|S_{[\Pi]}(X)\right|}{\left|\Gamma_{X}\right|} .
$$

Because $\Gamma_{X} \in[\Pi]$ implies both $S_{[\Pi]}(X)=\left\{\Gamma_{X}\right\}$ and $\left|\Gamma_{X}\right|=|\Pi|$, we have (cf. (1))

$$
\left|\mathscr{L}_{[\Pi]}\right|=|\Gamma| \frac{N_{[\Pi]}}{|\Pi|}+\left|\mathscr{C}_{[\Pi]}\right| .
$$

Equation (6) is now the crux of our counting approach: First, we compute $\left|\mathscr{L}_{[\Pi]}\right|$ nonconstructively. Second, we constructively enumerate the orbits $[X]$ with $|\Pi|<\left|\Gamma_{X}\right|$, which gives us $\left|\mathscr{C}_{[\Pi]}\right|$ via (4) and (5). Finally, we solve (6) for $N_{[\Pi]}$.

One further simplification is that the task of computing $\left|\mathscr{L}_{[\Pi]}\right|$ can be reduced from $[\Pi]$ to a representative group $\Pi \leq \Gamma$ in the conjugacy class. To this end, let

$$
\mathscr{L}_{\Pi}=\left\{X \in \mathscr{L}: \Pi \leq \Gamma_{X}\right\} .
$$

From (3), the fact that $\gamma \mathscr{L}_{\Pi}=\mathscr{L}_{\gamma \Pi \gamma^{-1}}$ for all $\gamma \in \Gamma$, and the Orbit-Stabilizer Theorem, we have

$$
\left|\mathscr{L}_{[\Pi]}\right|=\sum_{\Sigma \in[\Pi]}\left|\mathscr{L}_{\Sigma}\right|=|[\Pi]| \cdot\left|\mathscr{L}_{\Pi}\right|=\frac{|\Gamma|}{\left|\Gamma_{\Pi}\right|}\left|\mathscr{L}_{\Pi}\right| .
$$

We now proceed to apply this approach in the setting of Latin squares.

1.3. Main classes. We work with the standard triple system representation of Latin squares. To this end, let $R=\left\{r_{1}, r_{2}, \ldots, r_{n}\right\}, C=\left\{c_{1}, c_{2}, \ldots, c_{n}\right\}$, and $S=\left\{s_{1}, s_{2}, \ldots, s_{n}\right\}$ be three pairwise disjoint $n$-element sets. The intuition is that $R$ indexes the rows, $C$ indexes the columns, and $S$ is the set of symbols. We use design-theoretic terminology and call the elements of $R \cup C \cup S$ points and the sets $R, C, S$ point classes.

A Latin square of order $n$ can now be represented as a set of $n^{2}$ triples, where each triple $\left\{r_{i}, c_{j}, s_{k}\right\}$ indicates that the symbol $s_{k}$ occurs in row $r_{i}$, column $c_{j}$ of the array. Put otherwise, a Latin square (in triple system representation) is a set $\mathcal{L}$ of triples over $R \cup C \cup S$ such that (a) any two points from different point classes occur together in a unique triple; and (b) no triple contains two points from the same point class.

Assuming $R, C, S$ are fixed but arbitrary, denote by $\mathscr{L}$ the set of all Latin squares in triple system representation. A "Latin square" in what follows refers to an element of $\mathscr{L}$. Accordingly, it is convenient to assume in what follows that a "pair" (of points) refers to a set of two points from two different point classes, and a "triple" (of points) refers to a set of three points from three different point classes.

Let $\Gamma$ be the group consisting of all permutations of $R \cup C \cup S$ that fix the partition $\{R, C, S\}$ setwise. The structure of this group is a wreath product $\Gamma=$ $S_{n} \prec S_{3} \cong\left(S_{n}\right)^{3} \rtimes S_{3}$ with the three copies of $S_{n}$ permuting the points within each of the three point classes, and $S_{3}$ permuting the point classes. In particular, $|\Gamma|=3 !(n !)^{3}$. 
The orbits of $\Gamma$ on $\mathscr{L}$ are called main classes. Two Latin squares in the same main class are paratopic. The stabilizer of a Latin square in $\Gamma$ is called the autoparatopy group; the elements of the group are autoparatopisms.

1.4. Proof outline. The rest of this paper constitutes a proof of Theorem 1 Our approach is to determine $N_{i}$ for $i=2$ via nonconstructive techniques enabled by $\$ 1.2$ and for $i>2$ via constructive enumeration of main class representatives. Finally, we solve for $N_{1}$ using (1), with $|\mathscr{L}|$ given by [14].

We begin in 92 by establishing the necessary background on autoparatopisms of Latin squares. In $\$ 3$ we carry out a constructive enumeration of all main classes of Latin squares admitting an autoparatopy group of order at least 3 . In $\$ 4$ we carry out a nonconstructive enumeration of Latin squares admitting fixed autoparatopisms of order 2. Finally, in $\$$ we complete the proof of Theorem 1 by applying (11), (2), (4), (5), and (6) to the results of $\$ 3$ and $\$ 4$

\section{Groups of autoparatopisms}

2.1. Prime order. A group element $\alpha \in \Gamma$ of prime order $p$ is characterized up to conjugation in $\Gamma$ by: (a) the prime order $p$; (b) the number of points fixed in each of the point classes $R, C$, and $S$; and (c) the number of point classes $R, C, S$ fixed. Denote by $f_{R}, f_{C}$, and $f_{S}$ the number of points fixed by $\alpha$ in $R, C$, and $S$, respectively. Up to conjugacy we may assume $f_{R} \geq f_{C} \geq f_{S}$. Denote by $F$ the number of point classes $R, C, S$ fixed by $\alpha$.

We proceed to narrow down the possible types $\left(p, f_{R}, f_{C}, f_{S}, F\right)$. The results in the following two lemmata are well known; see, for example, [13, Theorem 1].

Lemma 2. The order $p$ divides $3-F$. For each parameter $f$ in $f_{R}, f_{C}, f_{S}$ it holds that $f=0$ or $p$ divides $n-f$; furthermore, the latter property must hold for at least $F$ of the parameters.

Proof. A cycle decomposition of $\alpha$ consists only of $p$-cycles and fixed points. So if a point class is fixed - there are $F$ such point classes - and contains $f$ fixed points, then $p$ divides $n-f$. Similarly, $p$ divides $3-F$.

Lemma 3. Any group of autoparatopisms of a Latin square that fixes points from at least two point classes has the property that the fixed points induce a subsquare. In particular, if $f_{R} \geq 1$ and $f_{C} \geq 1$ for a nonidentity autoparatopism, then $f_{R}=$ $f_{C}=f_{S} \leq n / 2$.

Proof. Consider the triples in a Latin square. If a triple has two fixed points relative to a group of autoparatopisms, then the third point must also be fixed; otherwise the two fixed points would occur in at least two triples, which is impossible. Thus, the number of triples with three fixed points is $f_{R} f_{C}=f_{R} f_{S}=f_{C} f_{S}$, from which we conclude $f_{R}=f_{C}=f_{S}$ when $f_{R}, f_{C} \geq 1$. Moreover, the triples with three fixed points induce a Latin square on the fixed points. The order of a proper subsquare in a Latin square of order $n$ is at most $n / 2$ [1, Theorem 1.42].

We must consider the prime orders $p=2,3,5,7,11$ for $n=11$. Combining Lemmata 2 and 3 , we can exclude all other types $\left(p, f_{R}, f_{C}, f_{S}, F\right)$ except those listed in Table 1. This table also displays data related to the constructive enumeration to be discussed in $\$ 3$. 
TABLE 1. Autoparatopisms of prime order

\begin{tabular}{rrrrrrrrr}
\hline Type & $p$ & $f_{R}$ & $f_{C}$ & $f_{S}$ & $F$ & $T$ & Seeds & $M_{T}$ \\
\hline 1 & 2 & 1 & 1 & 1 & 3 & - & - & - \\
2 & 2 & 3 & 3 & 3 & 3 & - & - & - \\
3 & 2 & 5 & 5 & 5 & 3 & - & - & - \\
4 & 2 & 1 & 0 & 0 & 1 & - & - & - \\
5 & 2 & 3 & 0 & 0 & 1 & - & - & - \\
6 & 2 & 5 & 0 & 0 & 1 & - & - & - \\
7 & 2 & 7 & 0 & 0 & 1 & - & - & - \\
8 & 2 & 9 & 0 & 0 & 1 & - & - & - \\
9 & 2 & 11 & 0 & 0 & 1 & - & - & - \\
10 & 3 & 2 & 2 & 2 & 3 & $\mathrm{f} 3$ & 13 & 4 \\
11 & 3 & 5 & 5 & 5 & 3 & $\mathrm{f} 3$ & 30 & 25 \\
12 & 3 & 0 & 0 & 0 & 0 & $\mathrm{c} 2 \mathrm{n}$ & 339638 & 165 \\
13 & 5 & 1 & 1 & 1 & 3 & $\mathrm{f} 3$ & 7 & 1 \\
14 & 7 & 4 & 4 & 4 & 3 & $\mathrm{f} 3$ & 6 & 16 \\
15 & 11 & 0 & 0 & 0 & 3 & $\mathrm{~m} 1$ & 28 & 33 \\
16 & 11 & 11 & 0 & 0 & 3 & $\mathrm{f} 1 \mathrm{~m} 1$ & 1 & 242 \\
\hline
\end{tabular}

2.2. Order four. We next determine the subgroups $\Pi \leq \Gamma$ of order 4 whose elements of order 2 belong to the conjugacy classes in Table 1 . We call such elements of order 2 eligible elements.

We first compute the conjugacy classes of elements of $\Gamma$ (there are 34048 classes) using standard algorithms and isolate the 374 classes of order 4. Amongst these we find 6 classes for which the squares of elements are eligible. We observe that the elements in each of these classes are conjugate to their inverses. Therefore, there are 6 classes of cyclic groups of order 4. They are listed as types 38-43 in Table 3 , with generator permutations (column "Generators") appearing in Table 4

We note that the conjugacy classes of eligible elements, as well as of elements of order 4 with eligible squares, are determined by their permutation cycle structure $1^{a_{1}} 2^{a_{2}} 4^{a_{4}}$ for $a_{1}+2 a_{2}+4 a_{4}=3 n$ and the number $F$ of point classes $R, C, S$ that they fix. Table 2 indicates these 16 conjugacy classes.

To classify elementary abelian subgroups of order 4, we note that each such subgroup must contain three elements of order 2, any two of which generate the group. We will first classify such generating pairs $(\alpha, \beta)$, where both $\alpha \in \Gamma$ and $\beta \in \Gamma$ are eligible, and $\beta$ is contained in the centralizer of $\alpha$, which we denote by $\Gamma_{\alpha}=\left\{\gamma \in \Gamma: \gamma \alpha \gamma^{-1}=\alpha\right\}$. Furthermore, $\alpha \beta$ must be eligible as well. Clearly, any two of the three elements $\alpha, \beta, \alpha \beta$ generate the same group. We therefore can assume, without loss of generality, that (in an arbitrary total order on the conjugacy classes of $\Gamma$, for example, the "Type" number in Table 2) the class of $\alpha$ is not larger than the class of $\beta$, which is not larger than the class of $\alpha \beta$. If this is fulfilled, we call the pair $(\alpha, \beta)$ valid.

Furthermore, we need to classify such pairs only up to conjugacy in $\Gamma$. For this, we may assume that $\alpha$ is the chosen representative of its conjugacy class and $\beta$ is chosen up to conjugacy by $\Gamma_{\alpha}$. We determine the representatives for $\beta$ by computing for each eligible $\alpha$ the conjugacy classes of $\Gamma_{\alpha}$ and identifying amongst 
TABLE 2. Autoparatopism types for order dividing 4

\begin{tabular}{rrr}
\hline Type & Cycles & $F$ \\
\hline 1 & $1^{3} 2^{15}$ & 3 \\
2 & $1^{9} 2^{12}$ & 3 \\
3 & $1^{15} 2^{9}$ & 3 \\
4 & $1^{1} 2^{16}$ & 1 \\
5 & $1^{3} 2^{15}$ & 1 \\
6 & $1^{5} 2^{14}$ & 1 \\
7 & $1^{7} 2^{13}$ & 1 \\
8 & $1^{9} 2^{12}$ & 1 \\
9 & $1^{11} 2^{11}$ & 1 \\
10 & $1^{3} 2^{3} 4^{6}$ & 3 \\
11 & $1^{5} 2^{2} 4^{6}$ & 3 \\
12 & $1^{7} 2^{1} 4^{6}$ & 3 \\
13 & $1^{9} 4^{6}$ & 3 \\
14 & $1^{1} 2^{4} 4^{6}$ & 1 \\
15 & $1^{3} 2^{3} 4^{6}$ & 1 \\
16 & $1^{33}$ & 3 \\
\hline
\end{tabular}

these the classes of eligible elements. For each such class representative $\beta$ we test whether $(\alpha, \beta)$ is valid, and collect the valid pairs in a list.

Because conjugacy of subgroups conjugates elements to elements, the lexicographic choice of generating pairs ensures that the only remaining potential conjugacy amongst subgroups generated by such pairs can happen if both $\alpha$ and $\beta$, or $\beta$ and $\alpha \beta$ are in the same class under $\Gamma$.

A classification of such pairs on the computer finds 37 valid pairs; none of the corresponding subgroups are conjugate, as verified by explicit conjugacy tests in $\Gamma$. These groups are listed as types 1-37 in Table 3, again generators appear in Table 4 .

Calculations were done in the system GAP [3].

Up to conjugacy in $\Gamma$, there are now exactly 43 groups $\Pi \leq \Gamma$ of order 4 whose elements of order 2 belong to the conjugacy classes in Table 1 .

It turns out that these groups are distinguished up to conjugacy in $\Gamma$ by the conjugacy classes of their elements (column "Elements") and the lengths $1^{a_{1}} 2^{a_{2}} 4^{a_{4}}$ for $a_{1}+2 a_{2}+4 a_{4}=3 n$ of their orbits on $R \cup C \cup S$ (column "Orbits").

We can rule out the following groups with combinatorial arguments.

Lemma 4. The groups of types 1, 3, 4, 5, 9, 10, 13, 16, 24, 27, 40, and 41 are not admitted by a Latin square of order 11.

Proof. Let $R=\{1,2, \ldots, 11\}, C=\{12,13, \ldots, 22\}$, and $S=\{23,24, \ldots, 33\}$.

Types 4, 10, 16, 27, 40, and 41: All point classes contain fixed points, but their number varies between point classes. This contradicts Lemma 3 
TABle 3. Candidate groups of order 4

\begin{tabular}{|c|c|c|c|c|c|c|}
\hline Type & Generators & Elements & Orbits & $T$ & Seeds & $M_{T}$ \\
\hline $1^{*}$ & $\gamma_{1}, \gamma_{2}$ & $3,6,9,16$ & $1^{5} 2^{8} 4^{3}$ & - & - & - \\
\hline 2 & $\gamma_{1}, \gamma_{3}$ & $3,4,7,16$ & $1^{1} 2^{10} 4^{3}$ & $\mathrm{f} 1 \mathrm{~m} 2$ & 102 & 11 \\
\hline $3^{*}$ & $\gamma_{1}, \gamma_{4}$ & $2,3,3,16$ & $1^{3} 2^{15}$ & - & - & - \\
\hline $4^{*}$ & $\gamma_{1}, \gamma_{5}$ & $2,3,3,16$ & $1^{7} 2^{9} 4^{2}$ & - & - & - \\
\hline $5^{*}$ & $\gamma_{1}, \gamma_{6}$ & $3,7,8,16$ & $1^{5} 2^{8} 4^{3}$ & - & - & - \\
\hline 6 & $\gamma_{1}, \gamma_{7}$ & $3,5,6,16$ & $1^{1} 2^{10} 4^{3}$ & $\mathrm{f} 1 \mathrm{~m} 2$ & 230 & 11 \\
\hline 7 & $\gamma_{1}, \gamma_{8}$ & $1,2,3,16$ & $1^{3} 2^{9} 4^{3}$ & f3 & 40 & 1 \\
\hline 8 & $\gamma_{1}, \gamma_{9}$ & $3,6,7,16$ & $1^{5} 2^{6} 4^{4}$ & $\mathrm{f} 3 \mathrm{n}$ & 80 & 10 \\
\hline $9 *$ & $\gamma_{1}, \gamma_{10}$ & $3,4,5,16$ & $1^{1} 2^{8} 4^{4}$ & - & - & - \\
\hline $10^{*}$ & $\gamma_{1}, \gamma_{11}$ & $2,3,3,16$ & $1^{5} 2^{12} 4^{1}$ & - & - & - \\
\hline 11 & $\gamma_{1}, \gamma_{12}$ & $3,5,8,16$ & $1^{3} 2^{9} 4^{3}$ & f3n & 80 & 1 \\
\hline 12 & $\gamma_{1}, \gamma_{13}$ & $3,6,7,16$ & $1^{3} 2^{9} 4^{3}$ & f3n & 80 & 1 \\
\hline $13^{*}$ & $\gamma_{1}, \gamma_{14}$ & $2,3,3,16$ & $1^{9} 2^{6} 4^{3}$ & - & - & - \\
\hline 14 & $\gamma_{1}, \gamma_{15}$ & $3,5,6,16$ & $1^{3} 2^{7} 4^{4}$ & $\mathrm{f} 3 \mathrm{n}$ & 80 & 1 \\
\hline 15 & $\gamma_{16}, \gamma_{17}$ & $2,2,2,16$ & $1^{9} 4^{6}$ & f3 & 12 & 9 \\
\hline $16^{*}$ & $\gamma_{16}, \gamma_{18}$ & $2,2,2,16$ & $1^{5} 2^{6} 4^{4}$ & - & - & - \\
\hline 17 & $\gamma_{16}, \gamma_{19}$ & $1,1,2,16$ & $1^{3} 2^{3} 4^{6}$ & f3 & 20 & 1 \\
\hline 18 & $\gamma_{16}, \gamma_{20}$ & $2,5,9,16$ & $1^{3} 2^{7} 4^{4}$ & $\mathrm{f} 3 \mathrm{n}$ & 127 & 1 \\
\hline 19 & $\gamma_{16}, \gamma_{21}$ & $2,7,7,16$ & $1^{3} 2^{7} 4^{4}$ & $\mathrm{f} 3 \mathrm{n}$ & 83 & 1 \\
\hline 20 & $\gamma_{16}, \gamma_{22}$ & $2,5,5,16$ & $1^{3} 2^{3} 4^{6}$ & $\mathrm{f} 3 \mathrm{n}$ & 83 & 1 \\
\hline 21 & $\gamma_{16}, \gamma_{23}$ & $2,6,8,16$ & $1^{3} 2^{7} 4^{4}$ & f3n & 127 & 1 \\
\hline 22 & $\gamma_{16}, \gamma_{24}$ & $2,4,8,16$ & $1^{1} 2^{8} 4^{4}$ & $\mathrm{f} 1 \mathrm{~m} 2$ & 608 & 11 \\
\hline 23 & $\gamma_{16}, \gamma_{25}$ & $2,6,6,16$ & $1^{1} 2^{8} 4^{4}$ & $\mathrm{f} 1 \mathrm{~m} 1$ & 2215 & 22 \\
\hline $24^{*}$ & $\gamma_{16}, \gamma_{26}$ & $2,4,4,16$ & $1^{1} 2^{4} 4^{6}$ & - & - & - \\
\hline 25 & $\gamma_{16}, \gamma_{27}$ & $2,5,7,16$ & $1^{1} 2^{8} 4^{4}$ & $\mathrm{f} 1 \mathrm{~m} 1$ & 2835 & 22 \\
\hline 26 & $\gamma_{16}, \gamma_{28}$ & $2,2,2,16$ & $1^{3} 2^{9} 4^{3}$ & f3 & 10 & 1 \\
\hline $27^{*}$ & $\gamma_{16}, \gamma_{29}$ & $2,2,2,16$ & $1^{7} 2^{3} 4^{5}$ & - & - & - \\
\hline 28 & $\gamma_{16}, \gamma_{30}$ & $2,5,7,16$ & $1^{3} 2^{5} 4^{5}$ & $\mathrm{f} 3 \mathrm{n}$ & 127 & 1 \\
\hline 29 & $\gamma_{16}, \gamma_{31}$ & $2,6,6,16$ & $1^{3} 2^{5} 4^{5}$ & $\mathrm{f} 3 \mathrm{n}$ & 83 & 1 \\
\hline 30 & $\gamma_{16}, \gamma_{32}$ & $2,4,6,16$ & $1^{1} 2^{6} 4^{5}$ & $\mathrm{f} 1 \mathrm{~m} 1$ & 2854 & 22 \\
\hline 31 & $\gamma_{16}, \gamma_{33}$ & $2,5,5,16$ & $1^{1} 2^{6} 4^{5}$ & $\mathrm{f} 1 \mathrm{~m} 1$ & 3351 & 22 \\
\hline 32 & $\gamma_{34}, \gamma_{35}$ & $1,4,9,16$ & $1^{1} 2^{6} 4^{5}$ & $\mathrm{f} 1 \mathrm{~m} 1$ & 194 & 22 \\
\hline 33 & $\gamma_{34}, \gamma_{36}$ & $1,6,7,16$ & $1^{1} 2^{6} 4^{5}$ & $\mathrm{f} 1 \mathrm{~m} 1$ & 2300 & 22 \\
\hline 34 & $\gamma_{34}, \gamma_{37}$ & $1,5,8,16$ & $1^{1} 2^{6} 4^{5}$ & $\mathrm{f} 1 \mathrm{~m} 1$ & 1083 & 22 \\
\hline 35 & $\gamma_{34}, \gamma_{38}$ & $1,4,7,16$ & $1^{1} 2^{4} 4^{6}$ & $\mathrm{f} 1 \mathrm{~m} 1$ & 1696 & 22 \\
\hline 36 & $\gamma_{34}, \gamma_{39}$ & $1,5,6,16$ & $1^{1} 2^{4} 4^{6}$ & $\mathrm{f} 1 \mathrm{~m} 1$ & 6406 & 22 \\
\hline 37 & $\gamma_{34}, \gamma_{40}$ & $1,4,5,16$ & $1^{1} 2^{2} 4^{7}$ & $\mathrm{f} 1 \mathrm{~m} 1$ & 1761 & 22 \\
\hline 38 & $\gamma_{41}$ & $2,13,13,16$ & $1^{9} 4^{6}$ & f3 & 20 & 9 \\
\hline 39 & $\gamma_{42}$ & $2,10,10,16$ & $1^{3} 2^{3} 4^{6}$ & f3 & 20 & 1 \\
\hline $40^{*}$ & $\gamma_{43}$ & $2,11,11,16$ & $1^{5} 2^{2} 4^{6}$ & - & - & - \\
\hline $41^{*}$ & $\gamma_{44}$ & $2,12,12,16$ & $1^{7} 2^{1} 4^{6}$ & - & - & - \\
\hline 42 & $\gamma_{45}$ & $2,15,15,16$ & $1^{3} 2^{3} 4^{6}$ & $\mathrm{f} 3 \mathrm{n}$ & 4 & 1 \\
\hline 43 & $\gamma_{46}$ & $2,14,14,16$ & $1^{1} 2^{4} 4^{6}$ & $\mathrm{f} 1 \mathrm{~m} 1$ & 242 & 22 \\
\hline
\end{tabular}

*) Excluded by Lemma 4 
TABle 4. Generators for Table 3

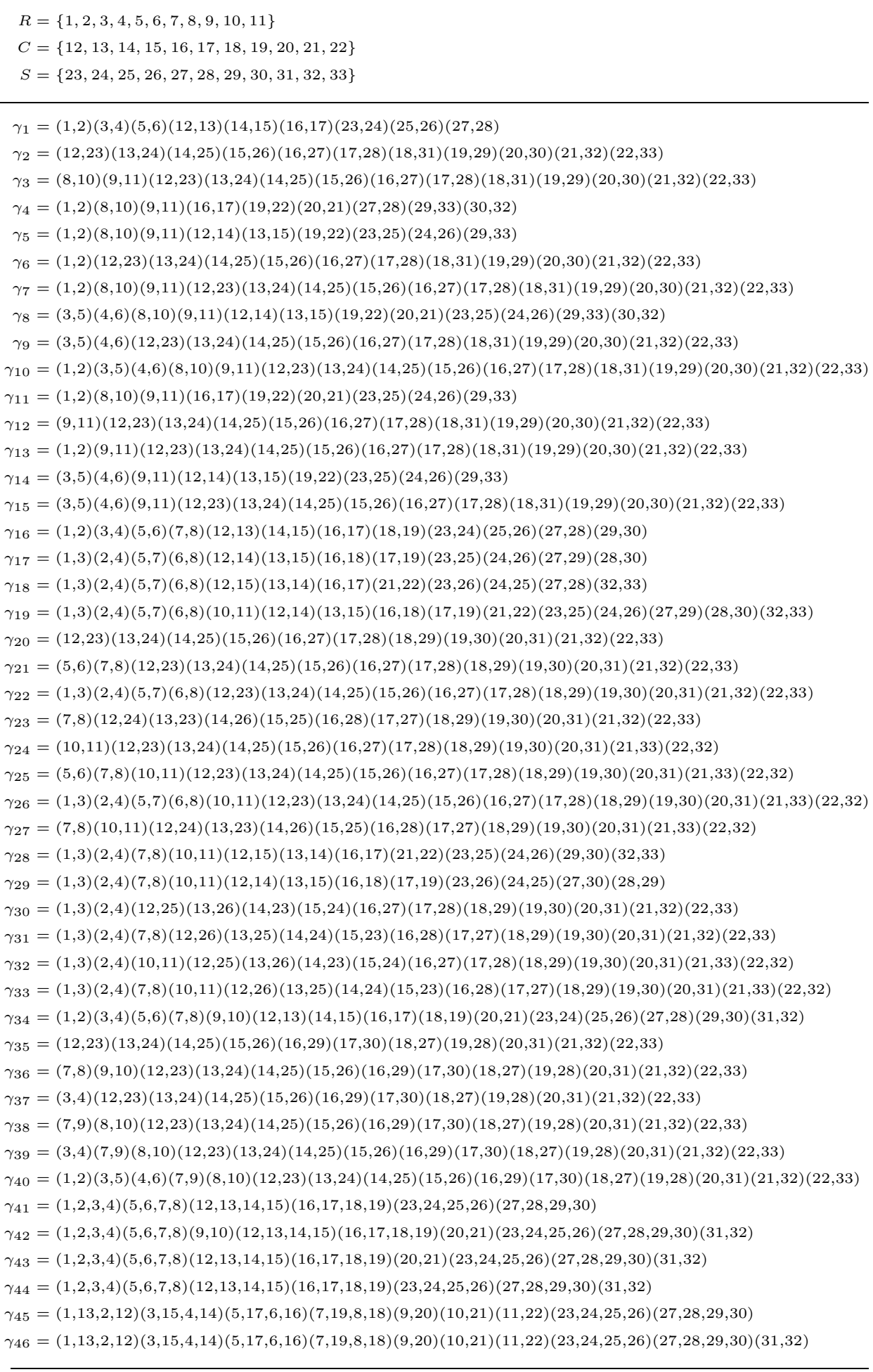


Type 1: Let

$$
\begin{array}{ll}
R_{1}=\{1,2,3,4,5,6\}, & R_{2}=\{7,8,9,10,11\}, \\
C_{1}=\{12,13,14,15,16,17\}, & C_{2}=\{18,19,20,21,22\}, \\
S_{1}=\{23,24,25,26,27,28\}, & S_{2}=\{29,30,31,32,33\} .
\end{array}
$$

Lemma 3 applied to $\gamma_{1}$ reveals that $R_{2} \cup C_{2} \cup S_{2}$ induces a $5 \times 5$ subsquare. Thus, there exists no triple with exactly two points from $R_{2} \cup C_{2} \cup S_{2}$.

Next consider pairs with one point from $C_{1}$ and one from $S_{1}$; there are $\left|C_{1}\right| \cdot\left|S_{1}\right|=$ 36 such pairs. The action of the group partitions these pairs combined with any points from $R$ into orbits, exactly six of which have length 4 ; for example, one such orbit is

$$
\left\{\{x, 12,25\},\{x, 14,23\},\left\{\gamma_{1}(x), 13,26\right\},\left\{\gamma_{1}(x), 15,24\right\}\right\} .
$$

As $\gamma_{1}(x)=x$ when $x \in R_{2}$, any such $x$ can be in at most one orbit (otherwise it would occur in at least $2 \cdot 4=8$ triples together with points from $C_{1}$, but $\left.\left|C_{1}\right|=6<8\right)$.

It follows that $x \in R_{2}$ in at most five of the above mentioned six orbits of length 4, whereby $x \in R_{1}$ in at least one of the orbits. Such an orbit with $x \in R_{1}$ contains two pairs $\{x, y\}$ with $y \in C_{1}$ (and recall that the third point of the triple is from $\left.S_{1}\right)$. Finally, consider how pairs $\{x, z\}$ with $z \in S_{2}$ are covered. The third point in the corresponding triple has to come from $C_{1}$ (by the initial comment). However, we have 5 points in $S_{2}$ to be combined with $x$ and $6-2=4$ points in $C_{1}$, which is not possible.

Type 3: It follows by Lemma 3 that there are $5 \times 5$ subsquares over $\{7,8,9,10,11\}$, $\{18,19,20,21,22\},\{29,30,31,32,33\}$ and $\{3,4,5,6,7\},\{12,13,14,15,18\},\{23,24$, $25,26,31\}$ for $\gamma_{1}$ and $\gamma_{4}$, respectively.

Consider the four triples containing point 3 and one point from $\{19,20$, $21,22\}$. By the given subsquare structure, the only possibilities for the third point of the triple are the points $\{27,28\}$, implying that $\{3,27\}$ or $\{3,28\}$ would occur in more than one triple.

Type 5: The proof for Type 1 is applicable as such, since the only difference between these cases is the additional transposition $(1,2)$ in generator $\gamma_{6}$, which leads to different orbits - for example, $\{1,12,25\},\{2,14,23\},\{2,13,26\},\{1,15,24\}$ instead of $\{1,12,25\},\{1,14,23\},\{2,13,26\},\{2,15,24\}$-but has no impact on the arguments of the proof.

Type 9: The only point fixed by $\gamma_{10}$ is 7 , which is then the only possible third point in triples containing the points in transpositions of $\gamma_{10}$ with one point each from $C$ and $S$. Since there are 11 such triples, the point 7 does not occur in any other triples.

By considering possible choices for $x$ in $\{x, 12,24\}$, we get up to symmetry four cases with the orbits

$$
\begin{aligned}
& \{\{1,12,24\},\{2,13,23\}\}, \\
& \{\{3,12,24\},\{4,13,23\},\{5,13,23\},\{6,12,24\}\}, \\
& \{\{8,12,24\},\{8,13,23\},\{10,13,23\},\{10,12,24\}\}, \text { and } \\
& \{\{7,12,24\},\{7,13,23\}\} .
\end{aligned}
$$

Because of the earlier argument, we cannot have $x=7$, and because pairs of points occur twice in two other cases, we must have $x \in\{1,2\}$. 
Repeated use of the previous reasoning gives that each of the pairs $\{12,24\}$, $\{13,23\},\{14,26\},\{15,25\},\{16,28\}$, and $\{17,27\}$ must occur in triples together with the points 1 and 2 (three times each). Now consider triples $\{1, x, y\}$ with $y \in\{29,30,31,32,33\}=S_{2}$. It follows from the subsquare implied by $\gamma_{1}$ that we cannot have $x \in\{18,19,20,21,22\}=C_{2}$. Now there are $\left|C \backslash C_{2}\right|=6$ possible choices for $x, 3$ of which already occur in triples together with the point 1 ; but $6-3=3<5=\left|S_{2}\right|$.

Type 13: By applying Lemma 3 to $\gamma_{1}$ and $\gamma_{1} \gamma_{14}$ we get that there are a $5 \times 5$ subsquare over $\{7,8,9,10,11\},\{18,19,20,21,22\},\{29,30,31,32,33\}$ and a $3 \times 3$ subsquare over $\{7,8,10\},\{18,20,21\},\{30,31,32\}$, respectively. But the latter is a subsquare of the former, and a $5 \times 5$ square cannot contain a $3 \times 3$ subsquare [1, Theorem 1.42].

Type 24: Analogously to the case of Type 9, we argue that, as the only point fixed by $\gamma_{26}$ is 9 , this point occurs exactly in triples with the other two points from transpositions of $\gamma_{26}$.

By considering possible choices for $x$ in $\{x, 12,24\}$, we get up to symmetry three cases with the orbits

$$
\begin{aligned}
& \{\{1,12,24\},\{3,13,23\},\{4,12,24\},\{2,13,23\}\}, \\
& \{\{10,12,24\},\{10,13,23\},\{11,13,23\},\{11,12,24\}\}, \text { and } \\
& \{\{9,12,24\},\{9,13,23\}\} .
\end{aligned}
$$

But because of the earlier argument, we cannot have $x=9$, and pairs of points occur twice in the other two cases.

We remark that the combinatorial proofs in Lemma 4 were triggered by computer searches showing nonexistence.

\section{Constructive enumeration}

3.1. Admissible groups. We recall the following corollary of the Sylow Theorems.

Theorem 5 ([16, Corollary 4.15]). Let $\Lambda$ be a finite group, let $p$ be a prime, and let $k$ be a positive integer. If $p^{k}$ divides $|\Lambda|$, then $\Lambda$ contains a subgroup of order $p^{k}$.

Let us say that a Latin square $\mathcal{L}$ admits $\Lambda \leq \Gamma$ as a group of autoparatopisms if $\Lambda \leq \Gamma_{\mathcal{L}}$. Combining Theorem 5 with the analysis in $₫ 2$, we have that every Latin square $\mathcal{L}$ of order 11 with $\left|\Gamma_{\mathcal{L}}\right| \geq 3$ admits at least one subgroup conjugate to: (a) a group of odd prime order in Table 1 or (b) a group of order 4 in Table 3 not excluded by Lemma 4. Let us call these groups the admissible groups.

3.2. The enumeration. We apply the framework in [5] to construct exactly one representative from each main class of Latin squares admitting at least one admissible group.

Associated with each conjugacy class $[\Pi]$ of admissible groups is a set of seeds, at least one of which is guaranteed to occur in every Latin square admitting $\Pi$.

The formal definition of a seed is as follows. Let $\Pi \leq \Gamma$ be an admissible group. Let $T \subseteq R \cup C \cup S$ be a set of points whose size and composition is determined by column " $T$ " in Tables 1 and 3 as follows:

$\mathrm{f} i$ : indicates $i$ points fixed by $\Pi$;

$\mathrm{m} i$ : indicates $i$ points moved by $\Pi$;

c $i$ : indicates $i$ points in same point class;

$\mathrm{n}$ : see item $(\mathrm{d})$ below. 
(For example, "f1m2" indicates that $T$ consists of 3 points, 1 point fixed by $\Pi$, and 2 points moved by $\Pi$.) Finally, let $\mathcal{S}$ be a union of $\Pi$-orbits of triples such that, referring to the elements of $\mathcal{S}$ as blocks,

(a) any pair occurs in at most one block;

(b) each point in $T$ occurs in exactly $n$ blocks;

(c) $T$ has nonempty intersection with at least one block on every $\Pi$-orbit on $\mathcal{S}$; and

(d) the set $T$ occurs in at least one block unless the composition of $T$ has the " $\mathrm{n}$ " indicator.

Each such tuple $(\Pi, T, \mathcal{S})$ is a seed associated with the conjugacy class $[\Pi]$.

Let $\gamma \in \Gamma$ act on a seed by $\gamma(\Pi, T, \mathcal{S})=\left(\gamma \Pi \gamma^{-1}, \gamma T, \gamma \mathcal{S}\right)$. The orbits of $\Gamma$ on the set of all seeds are the isomorphism classes of seeds. A Latin square $\mathcal{L}$ contains (or extends) a seed $(\Pi, T, \mathcal{S})$ if $\Pi \leq \Gamma_{\mathcal{L}}$ and $\mathcal{S} \subseteq \mathcal{L}$.

Given a seed $(\Pi, T, \mathcal{S})$, the task of finding all Latin squares that extend the seed is an instance of the exact cover problem. In particular, the task is to cover exactly once all uncovered pairs using $\Pi$-orbits of triples; each triple covers the pairs occurring in it.

Our constructive enumeration approach now proceeds as follows. First, we classify the seeds up to isomorphism using the algorithms described in [5]. The number of nonisomorphic seeds associated with each conjugacy class of admissible groups is given in column "Seeds" in Tables 1 and 3, Once the seeds have been classified, we use libexact 8 to find all extensions of each seed to a Latin square. As each extension $\mathcal{L}$ of a seed $(\Pi, T, \mathcal{S})$ is constructed, it is subjected to isomorph rejection tests developed in [5], which derive from the canonical augmentation technique of McKay [12. First, we identify a canonical $\Gamma_{\mathcal{L}}$-orbit of seeds contained in $\mathcal{L}$. If $(\Pi, T, \mathcal{S})$ does not occur in the identified orbit, we reject $\mathcal{L}$ from further consideration. Second, we test whether $\mathcal{L}$ is the lexicographic minimum of its $\Gamma_{(\Pi, T, \mathcal{S})}$-orbit. If not, we reject $\mathcal{L}$ from further consideration. Otherwise we accept $\mathcal{L}$ as the unique representative of its main class. See [6] for a detailed exposition of classification of combinatorial objects.

From an implementation perspective the present algorithm is almost identical to the one used in [5, 7]. Some additional implementation effort was required to work with the groups of order 4, and to implement associated correctness checks (to be discussed in (3.3). In particular, we must list all subgroups of order 4 in $\Gamma_{\mathcal{L}}$. This was implemented essentially using brute force, that is, by iterating over all elements of order 4, and all pairs of elements of order 2 that generate a group of order 4. (This strategy suffices because $\Gamma_{\mathcal{L}}$ is in most cases small; see Table 12.)

In the search we find 105670178597 Latin squares that extend the seeds. Among these, we find 1151666641 main classes. The number of main classes $N_{i}$ for each $i \geq 3$ is given later in Table 12

The search was distributed to a network of 155 Linux PCs using the batch system autoson [1]. In total the search consumed about 1.3 years of CPU time.

3.3. Correctness. We carry out a double counting check to gain confidence in the correctness of the constructive enumeration. The validation procedures considered in this section and later in $\$ 4.4$ are essential as, for example, number theoretic properties discussed in [18] and elsewhere are not applicable here. 
TABLE 5. Double count values for odd prime orders

\begin{tabular}{rrrrrrr}
\hline Type & $p$ & $f_{R}$ & $f_{C}$ & $f_{S}$ & $F$ & Count \\
\hline 10 & 3 & 2 & 2 & 2 & 3 & 88699187523260511795806208000000 \\
11 & 3 & 5 & 5 & 5 & 3 & 12309174115893617098752000000000 \\
12 & 3 & 0 & 0 & 0 & 0 & 19601984696323546934786654208000000 \\
13 & 5 & 1 & 1 & 1 & 3 & 710224896233056606617600000 \\
14 & 7 & 4 & 4 & 4 & 3 & 1186258276475008450560000000 \\
15 & 11 & 0 & 0 & 0 & 3 & 5968708870624483737600000 \\
16 & 11 & 11 & 0 & 0 & 3 & 38160882055721779200000 \\
\hline
\end{tabular}

Fix a conjugacy class of admissible groups $[\Pi]$. We compute in two different ways the total number of tuples $((\Sigma, T, \mathcal{S}), \mathcal{L})$ where $(\Sigma, T, \mathcal{S})$ is a seed contained in $\mathcal{L}$ and satisfying $\Sigma \in[\Pi]$.

For a Latin square $\mathcal{L}$, denote by seeds $[\Pi](\mathcal{L})$ the number of seeds associated with $[\Pi]$ contained in $\mathcal{L}$. For a seed $(\Sigma, T, \mathcal{S})$, denote by $\operatorname{ext}(\Sigma, T, \mathcal{S})$ the number of extensions of the seed into a Latin square. By the Orbit-Stabilizer Theorem, we have

$$
|\Gamma| \sum_{[\mathcal{L}]} \frac{\operatorname{seeds}_{[\Pi]}(\mathcal{L})}{\left|\Gamma_{\mathcal{L}}\right|}=|\Gamma| \sum_{[(\Sigma, T, \mathcal{S})]: \Sigma \in[\Pi]} \frac{\operatorname{ext}(\Sigma, T, \mathcal{S})}{\left|\Gamma_{(\Sigma, T, \mathcal{S}}\right|}
$$

where the sum on the left-hand side is over the main classes of Latin squares, and the sum on the right-hand side is over the isomorphism classes of seeds associated with $[\Pi]$.

The right-hand side of (9) is accumulated for each classified seed $(\Pi, T, \mathcal{S})$. In particular, $\operatorname{ext}(\Pi, T, \mathcal{S})$ is simply the number of solutions found in the exact cover search when extending $(\Pi, T, \mathcal{S})$.

The left-hand side of (9) is accumulated whenever a constructed $\mathcal{L}$ is accepted as the representative of its main class; that is, for every $[\Pi]$ we compute $\operatorname{seeds}_{[\Pi]}(\mathcal{L})$ and accumulate accordingly. To compute $\operatorname{seeds}_{[\Pi]}(\mathcal{L})$, we iterate over the subgroups $\Sigma \leq \Gamma_{\mathcal{L}}$ with $\Sigma \in[\Pi]$. For each such $\Sigma$, we accumulate seeds $\mathrm{s}_{[\Pi]}(\mathcal{L})$ by the value listed in column $M_{T}$ in Tables 1 and 3 . To justify this, first observe that whenever $\Sigma$ and $T$ are fixed, $\mathcal{L}$ determines $\mathcal{S}$ in a seed $(\Sigma, T, \mathcal{S})$ occurring in $\mathcal{L}$. In particular, $\mathcal{S}$ is the union of all $\Sigma$-orbits that contain a triple that has nonempty intersection with $T$. Now, whenever $\Sigma$ is fixed, $M_{T}$ counts the number of eligible sets $T$. The values $M_{T}$ can be determined using combinatorial arguments based on the structure of $\Pi$ and the size and composition constraints for $T$ in relation to $\Pi$.

Let us give two examples to illustrate the combinatorial arguments. First, Type 12 in Table 11 has $M_{T}=165$ because there are $3\left(\begin{array}{c}11 \\ 2\end{array}\right)=165$ ways to select two distinct points in a common point class. Second, Type 15 in Table 3 has $M_{T}=9$ because $T$ must consist of 3 points fixed by $\Sigma$ and must occur as a subset of (that is, must be equal to) a triple. There are 9 choices for such a $T$ by Lemma 3 , namely the 9 triples of the subsquare of order 3 induced by the points fixed by $\Sigma$.

The computed left-hand and right-hand sides of (9) agree for each [ח] in our constructive enumeration, which gives us confidence that the results are correct. We display the double count values for reference in Tables 5 and 6 . 
TABLE 6. Double count values for order 4

\begin{tabular}{rr}
\hline Type & Count \\
\hline 2 & 671631524180703313920000000 \\
6 & 2014894572542109941760000000 \\
7 & 3516906890255319171072000000 \\
8 & 54951670160239362048000000000 \\
11 & 1831722338674645401600000000 \\
12 & 5495167016023936204800000000 \\
14 & 21980668064095744819200000000 \\
15 & 307619449557019948744704000000 \\
17 & 39732092772896030588928000000 \\
18 & 13737917540059840512000000000 \\
19 & 268164150381968086794240000000 \\
20 & 65282584150364362113024000000 \\
21 & 179508789190115249356800000000 \\
22 & 16790788104517582848000000000 \\
23 & 1663496959090765967917056000000 \\
25 & 501843074867821516161024000000 \\
26 & 18539743730111373901824000000 \\
28 & 355683843723842644082688000000 \\
29 & 568713151711703904288768000000 \\
30 & 246354443069481975545856000000 \\
31 & 573841974259992911413248000000 \\
32 & 23802621216964125445324800000 \\
33 & 22878993544910822248022016000000 \\
34 & 1477320700587875009298432000000 \\
35 & 464500362123374411907072000000 \\
36 & 12596852214923599074557952000000 \\
37 & 61521447614952423555072000000 \\
38 & 919890958482406920683520000000 \\
39 & 39728022278810086932480000000 \\
42 & 45133638424943262695424000000 \\
43 & 2532319498770923774803968000000 \\
\hline &
\end{tabular}

\section{Nonconstructive EnUmeration}

4.1. One-factorizations with symmetry. Our objective in this section is to reduce the task of computing $\left|\mathscr{L}_{\Pi}\right|$ for subgroups $\Pi \leq \Gamma$ with $|\Pi|=2$ to the task of counting one-factorizations of a complete bipartite graph with forced symmetry.

We refer to [19] for basic graph-theoretic terminology. All graphs considered are undirected, loopless, and without parallel edges. A one-factor of a graph $G$ is a spanning 1-regular subgraph of $G$. A one-factorization of $G$ is a set $\mathcal{F}$ of one-factors of $G$ such that every edge of $G$ occurs in a unique one-factor in $\mathcal{F}$. Denote by $\operatorname{LF}(G)$ the number of distinct one-factorizations of $G$.

Let $K_{n, n}$ be the complete bipartite graph with vertex set $R \cup C$ and bipartition $\{R, C\}$. Let $\mathcal{F}$ be a one-factorization of $K_{n, n}$, and let $f: S \rightarrow \mathcal{F}$ be a bijection that "labels" the one-factors in $\mathcal{F}$ with elements of $S$.

Lemma 6. There is a one-to-one correspondence between the tuples $(\mathcal{F}, f)$ and the Latin squares in $\mathscr{L}$. 
Proof. Each triple $\left\{r_{i}, c_{j}, s_{k}\right\}$ in a Latin square $\mathcal{L}$ corresponds to the edge $\left\{r_{i}, c_{j}\right\}$ in the one-factor $f\left(s_{k}\right)$ in $\mathcal{F}$.

In particular, Lemma 6 implies

$$
|\mathscr{L}|=n ! \cdot \operatorname{LF}\left(K_{n, n}\right) .
$$

We now introduce forced symmetry into this setting, that is, we proceed to study $\mathscr{L}_{\Pi}$ in light of Lemma 6. Our task is fortuitously simplified by the assumption $|\Pi|=2$, which implies that $\Pi$ fixes at least one of the point classes $R, C, S$ setwise. Without loss of generality (up to conjugation of $\Pi$ in $\Gamma$ ), in what follows we will assume that $\Pi$ fixes $S$ setwise.

Let $\Phi$ be the group consisting of all permutations of $R \cup C$ that fix the partition $\{R, C\}$ setwise. Let $\Phi$ act on the set of spanning subgraphs of $K_{n, n}$ by permuting the vertices, and extend the action in the natural way to one-factorizations of such graphs.

Observe that each element $\pi \in \Pi$ can be restricted to $R \cup C$ because $\Pi$ fixes $S$ setwise. Let $\Delta$ be the restriction of $\Pi$ to $R \cup C$, and observe that $\Delta \leq \Phi$.

Consider now a Latin square $\mathcal{L}$ with $\Pi \leq \Gamma_{\mathcal{L}}$, and let $(\mathcal{F}, f)$ be the corresponding tuple given by Lemma 6. We clearly have $\Delta \leq \Phi_{\mathcal{F}}$. However, it is not the case that any tuple $(\mathcal{F}, f)$ with $\Delta \leq \Phi_{\mathcal{F}}$ corresponds to a Latin square with $\Pi \leq \Gamma_{\mathcal{L}}$. In particular, the action of $\Delta$ on $\mathcal{F}$ need not be "compatible" with the action of $\Pi$ on $S$. We proceed with a detailed analysis.

Let $\mathcal{F}$ be a one-factorization of $K_{n, n}$ with $\Delta \leq \Phi_{\mathcal{F}}$, and let $f: S \rightarrow \mathcal{F}$ be a bijection. For any $\delta \in \Delta$, define $\bar{\delta}$ as the permutation of $R \cup C \cup \mathcal{F}$ induced by the action of $\delta$ on $R \cup C$ and on $\mathcal{F}$. (We assume that $R \cup C$ and $\mathcal{F}$ are disjoint sets.) Note that $\bar{\delta}$ is well defined because $\delta \in \Delta \leq \Phi_{\mathcal{F}}$. Let $\bar{\Delta}=\{\bar{\delta}: \delta \in \Delta\}$. Extend $f$ by the identity mapping on $R \cup C$ to the mapping $\bar{f}: R \cup C \cup S \rightarrow R \cup C \cup \mathcal{F}$. Let $\bar{f}^{-1} \bar{\Delta} \bar{f}=\left\{\bar{f}^{-1} \bar{\delta} \bar{f}: \delta \in \Delta\right\}$, and observe that $\bar{f}^{-1} \bar{\Delta} \bar{f} \leq \Gamma$. We say that $(\mathcal{F}, f)$ agrees with $\Pi$ if $\bar{f}^{-1} \bar{\Delta} \bar{f}=\Pi$.

Lemma 7. The tuple $(\mathcal{F}, f)$ agrees with $\Pi$ if and only if the corresponding Latin square $\mathcal{L}$ satisfies $\Pi \leq \Gamma_{\mathcal{L}}$.

Proof. This follows from Lemma 6 because $\Pi$ fixes $S$ setwise.

We say that $\mathcal{F}$ is compatible with $\Pi$ if there exists an $f: S \rightarrow \mathcal{F}$ such that $(\mathcal{F}, f)$ agrees with $\Pi$. Denote by $\Psi$ the group of all permutations of $R \cup C \cup S$ that fix $R \cup C$ pointwise.

Lemma 8. Let $\mathcal{F}$ be compatible with $\Pi$. Then there are exactly $\left|\Psi_{\Pi}\right|$ bijections $f$ such that $(\mathcal{F}, f)$ agrees with $\Pi$.

Proof. Fix a reference bijection and establish a one-to-one correspondence between the set of all bijections and $\Psi_{\Pi}$.

Denote by $\operatorname{LF}\left(K_{n, n}, \Pi\right)$ the number of distinct one-factorizations of $K_{n, n}$ compatible with $\Pi$. From Lemmata 7 and 8 it thus follows that

$$
\left|\mathscr{L}_{\Pi}\right|=\left|\Psi_{\Pi}\right| \cdot \operatorname{LF}\left(K_{n, n}, \Pi\right) .
$$

The next three lemmata rely on our assumption that $\Pi$ has order 2 . 
Lemma 9. Suppose $\Pi$ restricted to $S$ has $a_{1}$ orbits of length 1 and $a_{2}$ orbits of length 2. Then

$$
\left|\Psi_{\Pi}\right|=a_{1} ! a_{2} ! 2^{a_{2}} .
$$

Proof. There are $a_{1} ! a_{2} ! 2^{a_{2}}$ permutations in $\Psi$ that fix the nonidentity element of $\Pi$ under conjugation.

Lemma 10. Let $\mathcal{F}$ be a one-factorization of $K_{n, n}$ with $\Delta \leq \Phi_{\mathcal{F}}$. Then $\mathcal{F}$ is compatible with $\Pi$ if and only if the action of $\Delta$ on $\mathcal{F}$ has an equal number of orbits of each length as the action of $\Pi$ on $S$.

Proof. The permutation of $S$ induced by the nonidentity element of $\Pi$ can be relabelled (by conjugation with an appropriate $f$ ) to the permutation of $\mathcal{F}$ induced by the nonidentity element of $\Delta$ if and only if there are an equal number of cycles of each length.

Let $G$ be a spanning subgraph of $K_{n, n}$. We say that a one-factorization $\mathcal{F}$ of $G$ is a $(\Delta, \vec{a})$-factorization if (a) $\Delta \leq \Phi_{\mathcal{F}}$; and (b) for each $i=1,2$, the action of $\Delta$ on $\mathcal{F}$ has $a_{i}$ orbits of length $i$ with $\vec{a}=\left(a_{1}, a_{2}\right)$. Denote by $\operatorname{LF}(G, \Delta, \vec{a})$ the number of distinct $(\Delta, \vec{a})$-factorizations of $G$.

Lemma 11. Suppose $\Pi$ restricted to $S$ has $a_{1}$ orbits of length 1 and $a_{2}$ orbits of length 2. Then

$$
\operatorname{LF}\left(K_{n, n}, \Pi\right)=\operatorname{LF}\left(K_{n, n}, \Delta, \vec{a}\right) .
$$

Proof. This is immediate from Lemma 10 .

We have now reduced the task of computing $\left|\mathscr{L}_{\Pi}\right|$ via (10), (11), and (12) to computing $\operatorname{LF}\left(K_{n, n}, \Delta, \vec{a}\right)$.

We employ two different methods to compute the values $\operatorname{LF}\left(K_{n, n}, \Delta, \vec{a}\right)$ for the conjugacy classes of order 2 in Table 1. The first method stems from a recursion over regular spanning subgraphs of $K_{n, n}$ that has evolved over a period of more than half a century into the form presented by McKay and Wanless [14. The second method extends a "forward accumulation" technique used in [7] to count the number of distinct one-factorizations of $K_{14}$.

Our present contribution is to modify both methods to take into account the symmetry forced by $\Delta$ and the required structure $\vec{a}=\left(a_{1}, a_{2}\right)$ for the lengths of the $\Delta$-orbits of one-factors.

4.2. Backward recursion. For convenience in what follows, let us abbreviate $\vec{e}_{1}=(1,0)$ and $\vec{e}_{2}=(0,1)$. We say that a spanning subgraph $G$ of $K_{n, n}$ is $(\Delta, \vec{a})$-factorizable if $G$ has at least one $(\Delta, \vec{a})$-factorization. Observe that a $(\Delta, \vec{a})$ factorizable graph is necessarily $k$-regular with $k=\sum_{i} i a_{i}$. Furthermore, each $(\Delta, \vec{a})$-factorizable graph decomposes into an edge-disjoint union consisting of $a_{1}$ $\left(\Delta, \vec{e}_{1}\right)$-factorizable and $a_{2}\left(\Delta, \vec{e}_{2}\right)$-factorizable graphs. Denote by $\mathcal{I}(G, \Delta, i)$ the set of all $\left(\Delta, \vec{e}_{i}\right)$-factorizable spanning subgraphs of $G$.

Counting in two different ways the number of distinct $(\Delta, \vec{a})$-factorizations of $G$ with one individualized $\Delta$-orbit consisting of $i$ one-factors, we obtain, for $a_{i} \geq 1$,

$$
a_{i} \cdot \operatorname{LF}(G, \Delta, \vec{a})=\sum_{F \in \mathcal{I}(G, \Delta, i)} \operatorname{LF}\left(F, \Delta, \vec{e}_{i}\right) \cdot \operatorname{LF}\left(G-F, \Delta, \vec{a}-\vec{e}_{i}\right) .
$$


In particular, if we know (for example, by recursion) the value $\operatorname{LF}\left(H, \Delta, \vec{a}-\vec{e}_{i}\right)$ for all $\left(\Delta, \vec{a}-\vec{e}_{i}\right)$-factorizable $H$, we can use (13) to compute $\operatorname{LF}(G, \Delta, \vec{a})$ for all $(\Delta, \vec{a})$-factorizable $G$. Furthermore, $\operatorname{LF}(G, \Delta, \vec{a})$ needs to be computed for only one representative $(G, \Delta)$ chosen from the $\Phi$-orbit $[(G, \Delta)]$. Here $\Phi$ acts on $G$ by permutation of vertices and on $\Delta$ by conjugation.

This is analogous to the method (without the forced $\Delta$ and $\vec{a}$ ) used in 13 and other earlier studies on counting Latin squares.

4.3. Forward accumulation. We can visit every $(\Delta, \vec{a})$-factorizable orbit $[(G, \Delta)]$ with $a_{i} \geq 1$ by the following procedure: For each $\left(\Delta, \vec{a}-\vec{e}_{i}\right)$-factorizable orbit $[(H, \Delta)]$, consider exactly one tuple $(H, \Delta)$ from the orbit; for each such tuple $(H, \Delta)$, consider each graph $F \in \mathcal{I}\left(K_{n, n}-H, \Delta, i\right)$; for each tuple $(H, F, \Delta)$, visit the $(\Delta, \vec{a})$-factorizable orbit $[(H \cup F, \Delta)]$.

To compute the value $\operatorname{LF}(G, \Delta, \vec{a})$ for each visited orbit $[(G, \Delta)]$, we associate with $[(G, \Delta)]$ an accumulator variable $x_{[(G, \Delta)]}$ that is initially set to zero and incremented whenever $[(G, \Delta)]$ is visited. Our objective is to have the value $a_{i}$. $\operatorname{LF}(G, \Delta, \vec{a})$ in $x_{[(G, \Delta)]}$ when the procedure terminates.

We proceed to analyze the visiting procedure, with the objective of determining an appropriate increment to the counter variable on each visit. The following two lemmata are immediate consequences of the Orbit-Stabilizer Theorem (cf. [7. Lemmata 1,2]).

Lemma 12. Any tuple $(G, \Delta)$ in the orbit $[(H \cup F, \Delta)]$ admits exactly

$$
\sigma(H, F, \Delta)=\frac{\left|\Phi_{(H \cup F, \Delta)}\right|}{\left|\Phi_{(H, F, \Delta)}\right|}
$$

decompositions $G=H^{\prime} \cup F^{\prime}$ into tuples $\left(H^{\prime}, F^{\prime}, \Delta\right)$ in the orbit $[(H, F, \Delta)]$.

Lemma 13. The procedure visits an orbit $[(H \cup F, \Delta)]$ exactly

$$
\tau(H, F, \Delta)=\frac{\left|\Phi_{(H, \Delta)}\right|}{\left|\Phi_{(H, F, \Delta)}\right|}
$$

times via tuples $\left(H^{\prime}, F^{\prime}, \Delta\right)$ in the orbit $[(H, F, \Delta)]$.

It now follows from Lemma 12 and (13) that, for $a_{i} \geq 1$,

$$
\begin{aligned}
a_{i} \cdot \operatorname{LF}(G, \Delta, \vec{a}) & =\sum_{(H, F, \Delta): H \cup F=G} \operatorname{LF}\left(F, \Delta, \vec{e}_{i}\right) \cdot \operatorname{LF}\left(H, \Delta, \vec{a}-\vec{e}_{i}\right) \\
& =\sum_{[(H, F, \Delta)]: H \cup F=G} \sigma(H, F, \Delta) \cdot \operatorname{LF}\left(F, \Delta, \vec{e}_{i}\right) \cdot \operatorname{LF}\left(H, \Delta, \vec{a}-\vec{e}_{i}\right) .
\end{aligned}
$$

This observation enables us to accumulate the value $a_{i} \cdot \operatorname{LF}(G, \Delta, \vec{a})$ to $x_{[(G, \Delta)]}$. Namely, each time $[(G, \Delta)]$ is visited via a tuple $(H, F, \Delta)$, we increment $x_{[G, \Delta)]}$ by the rule

$$
x_{[(G, \Delta)]} \leftarrow x_{[(G, \Delta)]}+\frac{\sigma(H, F, \Delta)}{\tau(H, F, \Delta)} \cdot \operatorname{LF}\left(F, \Delta, \vec{e}_{i}\right) \cdot \operatorname{LF}\left(H, \Delta, \vec{a}-\vec{e}_{i}\right) .
$$

Equivalently, for each tuple $(H, F, \Delta)$ considered by the visiting procedure, we apply the rule

$$
x_{[(H \cup F, \Delta)]} \leftarrow x_{[(H \cup F, \Delta)]}+\frac{\left|\Phi_{(H \cup F, \Delta)}\right|}{\left|\Phi_{(H, \Delta)}\right|} \cdot \operatorname{LF}\left(F, \Delta, \vec{e}_{i}\right) \cdot \operatorname{LF}\left(H, \Delta, \vec{a}-\vec{e}_{i}\right) .
$$


Analogously to [7, Lemma 3], we have the following result.

Lemma 14. The total accumulation to $x_{[(G, \Delta)]}$ is $a_{i} \cdot \operatorname{LF}(G, \Delta, \vec{a})$.

Proof. By Lemma 13 and (15), the total accumulation to $x_{[(G, \Delta)]}$ from an orbit $[(H, F, \Delta)]$ satisfying $G=H \cup F$ is $\sigma(H, F, \Delta) \cdot \operatorname{LF}\left(F, \Delta, \vec{e}_{i}\right) \cdot \operatorname{LF}\left(H, \Delta, \vec{a}-\vec{e}_{i}\right)$. Taking the sum over all such classes, the claim follows by (14).

4.4. Implementation and correctness. Associated with each graph $F$ in 4.2 and 4.3 there are exactly $\operatorname{LF}\left(F, \Delta, \vec{e}_{i}\right)$ distinct $\Delta$-orbits (of length $i$ ) of one-factors; that is, the distinct $\left(\Delta, \vec{e}_{i}\right)$-factorizations of $F$. From an implementation perspective it is convenient to iterate over these $\Delta$-orbits directly instead of the underlying graphs $F$. In particular, we achieve the required accumulation in (13) and (16) without computing the values $\operatorname{LF}\left(F, \Delta, \vec{e}_{i}\right)$ explicitly.

Starting with the empty spanning subgraph of $K_{n, n}$, we use the forward accumulation method to compute the values $\operatorname{LF}(G, \Delta, \vec{a})$ for all $(\Delta, \vec{a})$-factorizable orbits $[(G, \Delta)]$. We carry out the forward accumulation in two different ways. First, we accumulate the $\Delta$-orbits in order of increasing length; that is, we first add all $\Delta$ orbits of length 1 , and then the $\Delta$-orbits of length 2 . Second, as a consistency check, we add the orbits in order of decreasing length.

In addition to the forward accumulation method, we verify the results using the recursion (13), that is, when we have accumulated the value $\operatorname{LF}(G, \Delta, \vec{a})$ by addition of orbits of length $i$, we apply (13) to check the result using the values $\operatorname{LF}\left(H, \Delta, \vec{a}-\vec{e}_{i}\right)$.

The two different orders of adding orbits enable yet another way to cross-check the results (cf. [2] and [7]). Namely,

$$
\operatorname{LF}\left(K_{n, n}, \Delta, \vec{a}+\vec{b}\right)=\frac{\sum_{[(G, \Delta)]} \frac{\left|\Phi_{\Delta}\right|}{\left|\Phi_{(G, \Delta)}\right|} \cdot \operatorname{LF}(G, \Delta, \vec{a}) \cdot \operatorname{LF}\left(K_{n, n}-G, \Delta, \vec{b}\right)}{\left(\begin{array}{c}
a_{1}+b_{1} \\
a_{1}
\end{array}\right)\left(\begin{array}{c}
a_{2}+b_{2} \\
a_{2}
\end{array}\right)}
$$

holds for every nonnegative $\vec{a}=\left(a_{1}, a_{2}\right)$ and $\vec{b}=\left(b_{1}, b_{2}\right)$ with $\sum_{i} i\left(a_{i}+b_{i}\right)=n$, where the sum is over all $(\Delta, \vec{a})$-factorizable orbits $[(G, \Delta)]$. Due to the two different orders of adding orbits, we cannot utilise all possible decompositions $\vec{a}+\vec{b}$ for checking, however. For example, if we add orbits in the orders 1,1,1,2,2,2,2 and $2,2,2,2,1,1,1$, then we can use eight different decompositions in (17).

The approaches were implemented using the $\mathrm{C}$ programming language with the help of three software libraries: nauty [10] for isomorphism testing, the GNU Multiple Precision Arithmetic Library 4] for handling large integers (and intermediate rationals), and libexact [8] for simplifying the search for $\Delta$-orbits of one-factors. The number of orbits $[(G, \Delta)]$ at any step of the search never exceeded 200000, so memory requirement was not a major issue of the search (compare with [7, 13]). The computations took about 20 days on a contemporary Linux PC.

4.5. Results. The numbers of $(\Delta, \vec{a})$-factorizable orbits $[(G, \Delta)]$ are shown in Tables 7 and 8 for situations when the one-factor orbits are appended in order of increasing and decreasing length $i$, respectively. The leftmost column contains the number of one-factor orbits $\sum_{i} a_{i}$, and there is one column for each of the conjugacy classes of order 2 in Table 1.

The second column of Table 10 summarizes the results of the search, which enable us to determine the values $\left|\mathscr{L}_{[\Pi]}\right|$ in the rightmost column of Table 10 via 
the combination of (8), (10), (11), and (12). That is,

$$
\left|\mathscr{L}_{[\Pi]}\right|=\frac{|\Gamma|}{\left|\Gamma_{\Pi}\right|} \cdot a_{1} ! a_{2} ! 2^{a_{2}} \cdot \operatorname{LF}\left(K_{11,11}, \Delta,\left(a_{1}, a_{2}\right)\right),
$$

where $|\Gamma|=3 !(11 !)^{3}$ and the values $\left|\Gamma_{\Pi}\right|$ are displayed in Table 9, these are derived by considering the number of permutations in $\Gamma$ that fix the nonidentity element of $\Pi$ under conjugation.

TABLE 7. Number of $(\Delta, \vec{a})$-factorizable orbits, increasing length

\begin{tabular}{crrrrrrrrr}
\hline$\sum_{i} a_{i} \backslash$ Type & 1 & 2 & 3 & 4 & 5 & 6 & 7 & 8 & 9 \\
\hline 0 & 1 & 1 & 1 & 1 & 1 & 1 & 1 & 1 & 1 \\
1 & 1 & 1 & 1 & 6 & 6 & 6 & 6 & 6 & 6 \\
2 & 304 & 10 & 10 & 1089 & 22 & 22 & 22 & 22 & 22 \\
3 & 34792 & 16 & 10 & 170321 & 1365 & 1365 & 1365 & 1365 & 1365 \\
4 & 8530 & 949 & 5 & 33851 & 184239 & 30429 & 30429 & 30429 & 30429 \\
5 & 20 & 1464 & 1 & 46 & 35286 & 146085 & 146085 & 146085 & 146085 \\
6 & 1 & 11 & 1 & 1 & 49 & 32039 & 125763 & 125763 & 125763 \\
7 & & 1 & 1 & & 1 & 45 & 19567 & 19567 & 19567 \\
8 & & & 1 & & & 1 & 36 & 630 & 630 \\
9 & & & & & & & 1 & 21 & 21 \\
10 & & & & & & & & 1 & 1 \\
11 & & & & & & & & & 1 \\
\hline
\end{tabular}

TABLE 8. Number of $(\Delta, \vec{a})$-factorizable orbits, decreasing length

\begin{tabular}{crrrrrrrrr}
\hline$\sum_{i} a_{i} \backslash$ Type & 1 & 2 & 3 & 4 & 5 & 6 & 7 & 8 & 9 \\
\hline 0 & 1 & 1 & 1 & 1 & 1 & 1 & 1 & 1 & 1 \\
1 & 5 & 6 & 1 & 6 & 6 & 6 & 6 & 6 & 6 \\
2 & 8528 & 1464 & 3 & 266 & 266 & 266 & 266 & 1089 & 22 \\
3 & 34792 & 1182 & 1 & 266 & 266 & 266 & 170321 & 35186 & 1365 \\
4 & 319 & 16 & 5 & 6 & 6 & 33851 & 187719 & 184239 & 30429 \\
5 & 1 & 10 & 10 & 1 & 46 & 1524 & 35286 & 178732 & 146085 \\
6 & 1 & 1 & 10 & 1 & 5 & 49 & 1475 & 32039 & 125763 \\
7 & & 1 & 1 & & 1 & 4 & 45 & 1220 & 19567 \\
8 & & & 1 & & & 1 & 3 & 36 & 630 \\
9 & & & & & & & 1 & 2 & 21 \\
10 & & & & & & & & 1 & 1 \\
11 & & & & & & & & & 1 \\
\hline
\end{tabular}


TABLE 9. $\left|\Gamma_{\Pi}\right|$ for conjugacy classes with $|\Pi|=2$

\begin{tabular}{rrr}
\hline Type & & $\left|\Gamma_{\Pi}\right|$ \\
\hline 1 & $\left(1 ! \cdot 5 ! \cdot 2^{5}\right)^{3} \cdot 3 !=$ & 339738624000 \\
2 & $\left(3 ! \cdot 4 ! \cdot 2^{4}\right)^{3} \cdot 3 !=$ & 73383542784 \\
3 & $\left(5 ! \cdot 3 ! \cdot 2^{3}\right)^{3} \cdot 3 !=$ & 1146617856000 \\
4 & $1 ! \cdot 5 ! \cdot 2^{5} \cdot 11 ! \cdot 2=$ & 306561024000 \\
5 & $3 ! \cdot 4 ! \cdot 2^{4} \cdot 11 ! \cdot 2=$ & 183936614400 \\
6 & $5 ! \cdot 3 ! \cdot 2^{3} \cdot 11 ! \cdot 2=$ & 459841536000 \\
7 & $7 ! \cdot 2 ! \cdot 2^{2} \cdot 11 ! \cdot 2=$ & 3218890752000 \\
8 & $9 ! \cdot 1 ! \cdot 2^{1} \cdot 11 ! \cdot 2=$ & 57940033536000 \\
9 & $11 ! \cdot 0 ! \cdot 2^{0} \cdot 11 ! \cdot 2=3186701844480000$ \\
\hline
\end{tabular}

TABLE 10. $\left|\mathscr{L}_{[\Pi]}\right|$ for conjugacy classes with $|\Pi|=2$

\begin{tabular}{rrr}
\hline Type & $\operatorname{LF}\left(K_{11,11}, \Delta, \vec{a}\right)$ & $\left|\mathscr{L}_{[\Pi]}\right|$ \\
\hline 1 & 157811617463135109120 & 680681075601465561779007809126400000 \\
2 & 16482860057738870784 & 197485300889612684060962848768000000 \\
3 & 57076088832000000 & 109414881030165485322240000000000 \\
4 & 81867724734136320 & 391332044120479692845639270400000 \\
5 & 91614680894074060800 & 437923008879888637579625496576000000 \\
6 & 984580848455978188800 & 4706348408721523581497414516736000000 \\
7 & 588014650532826316800 & 2810741057151330357801067216896000000 \\
8 & 39448015149028147200 & 188563593954724468142515421184000000 \\
9 & 252282619805368320 & 1205924234796020705924638310400000 \\
\hline
\end{tabular}

\section{Number of Equivalence Classes}

5.1. Main classes. As a result of the computations described in 33 and we have a constructive enumeration for all main classes with an autoparatopy group of order at least 3. The numbers of such main classes are given in Table 12 for $i \geq 3$. Table 10 enables us to solve the number of main classes with an autoparatopy group of order 2. Indeed, we compute $\left|\mathscr{C}_{[\Pi]}\right|$ by direct summation over the constructed main classes (5), and use (6) together with Table 10 to arrive at Table 11.

Table 11 and Table 12 (for $i \geq 3$ ) give

$$
|\Gamma| \sum_{i \geq 2} \frac{N_{i}}{i}=9023583561995938862980803959193600000 .
$$

The number of distinct Latin squares of order 11 is [14]

$$
\begin{aligned}
|\mathscr{L}| & =11 ! \cdot 10 ! \cdot 5363937773277371298119673540771840 \\
& =776966836171770144107444346734230682311065600000 .
\end{aligned}
$$

Solving for $N_{1}$ in (1) using (18) and (19), we obtain

$$
N_{1}=2036029552535590421717241
$$

and hence

$$
\sum_{i} N_{i}=2036029552582883134196099 .
$$


TABLE 11. $\left|\mathscr{C}_{[\Pi]}\right|$ and $N_{[\Pi]}$ for conjugacy classes with $|\Pi|=2$

\begin{tabular}{rrr}
\hline Type & $\left|\mathscr{C}_{[\Pi]}\right|$ & $N_{[\Pi]}$ \\
\hline 1 & 1788612130139530711950950400000 & 3567419044431 \\
2 & 2090765465832065147338752000000 & 1035003382971 \\
3 & 40157841013697699905536000000 & 573229534 \\
4 & 37761778205327560959590400000 & 2050761205 \\
5 & 1253232559784675856089088000000 & 2295134347303 \\
6 & 3238510153755989660663808000000 & 24665809156818 \\
7 & 2028400471919270993854464000000 & 14730996126592 \\
8 & 252164628166875895037952000000 & 988254629517 \\
9 & 14823428114332518344294400000 & 6320133846 \\
\hline$N_{2}$ & & 47291560812217 \\
\hline
\end{tabular}

TABLE 12. Main classes of Latin squares of order 11

\begin{tabular}{|c|c|c|c|}
\hline$i$ & $N_{i}$ & $i$ & $N_{i}$ \\
\hline 1 & 2036029552535590421717241 & 24 & 274 \\
\hline 2 & 47291560812217 & 27 & 27 \\
\hline 3 & 1111651266 & 30 & 4 \\
\hline 4 & 39004721 & 32 & 16 \\
\hline 5 & 9131 & 36 & 86 \\
\hline 6 & 960771 & 40 & 4 \\
\hline 7 & 1294 & 42 & 5 \\
\hline 8 & 30390 & 48 & 9 \\
\hline 9 & 2636 & 54 & 18 \\
\hline 10 & 323 & 60 & 1 \\
\hline 11 & 3 & 63 & 3 \\
\hline 12 & 4191 & 72 & 14 \\
\hline 14 & 105 & 108 & 5 \\
\hline 15 & 4 & 110 & 2 \\
\hline 16 & 631 & 120 & 1 \\
\hline 18 & 625 & 162 & 1 \\
\hline 20 & 37 & 324 & 1 \\
\hline 21 & 37 & 7260 & 1 \\
\hline 22 & 4 & $\sum_{i} N_{i}$ & 2036029552582883134196099 \\
\hline
\end{tabular}

Table 12 gives the number $N_{i}$ of main classes for each order $i$ of the autoparatopy group.

5.2. One-factorizations and isotopy classes. For a Latin square $\mathcal{L}$, denote by $\operatorname{Par}(\mathcal{L})$ the autoparatopy group of $\mathcal{L}$, and denote by $\operatorname{Is}(\mathcal{L})$ the subgroup of $\operatorname{Par}(\mathcal{L})$ that fixes each of the point classes $R, C, S$ setwise. This group is called the autotopy group of $\mathcal{L}$, and its elements are called autotopisms. Let $\operatorname{Ty}(\mathcal{L})=3,2,1,1$ when $|\operatorname{Par}(\mathcal{L})| /|\operatorname{Is}(\mathcal{L})|=1,2,3,6$, respectively. Observe that these quantities are independent of the main class representative $\mathcal{L}$ because $\operatorname{Is}(\mathcal{L})$ is a normal subgroup of $\operatorname{Par}(\mathcal{L})$. 
TABLE 13. Orders of autoparatopy and autotopy groups

\begin{tabular}{|c|c|c|c|c|c|}
\hline |Par| & $|\mathrm{Is}|$ & Main classes & |Par| & $|\mathrm{Is}|$ & Main classes \\
\hline 1 & 1 & 2036029552535590421717241 & 18 & 18 & 8 \\
\hline 2 & 1 & 42688565155281 & 20 & 10 & 22 \\
\hline 2 & 2 & 4602995656936 & 20 & 20 & 15 \\
\hline 3 & 1 & 933551378 & 21 & 7 & 32 \\
\hline 3 & 3 & 178099888 & 21 & 21 & 5 \\
\hline 4 & 2 & 36528967 & 22 & 11 & 2 \\
\hline 4 & 4 & 2475754 & 22 & 22 & 2 \\
\hline 5 & 5 & 9131 & 24 & 4 & 213 \\
\hline 6 & 1 & 742503 & 24 & 8 & 6 \\
\hline 6 & 2 & 28147 & 24 & 12 & 55 \\
\hline 6 & 3 & 164658 & 27 & 9 & 27 \\
\hline 6 & 6 & 25463 & 30 & 5 & 2 \\
\hline 7 & 7 & 1294 & 30 & 10 & 2 \\
\hline 8 & 4 & 27705 & 32 & 16 & 16 \\
\hline 8 & 8 & 2685 & 36 & 6 & 70 \\
\hline 9 & 3 & 2125 & 36 & 12 & 10 \\
\hline 9 & 9 & 511 & 36 & 18 & 6 \\
\hline 10 & 5 & 275 & 40 & 20 & 4 \\
\hline 10 & 10 & 48 & 42 & 14 & 2 \\
\hline 11 & 11 & 3 & 42 & 21 & 3 \\
\hline 12 & 2 & 2201 & 48 & 8 & 9 \\
\hline 12 & 4 & 400 & 54 & 9 & 17 \\
\hline 12 & 6 & 1470 & 54 & 27 & 1 \\
\hline 12 & 12 & 120 & 60 & 10 & 1 \\
\hline 14 & 7 & 79 & 63 & 21 & 3 \\
\hline 14 & 14 & 26 & 72 & 12 & 14 \\
\hline 15 & 5 & 4 & 108 & 18 & 5 \\
\hline 16 & 8 & 607 & 110 & 55 & 2 \\
\hline 16 & 16 & 24 & 120 & 60 & 1 \\
\hline 18 & 3 & 486 & 162 & 27 & 1 \\
\hline 18 & 6 & 35 & 324 & 54 & 1 \\
\hline 18 & 9 & 96 & 7260 & 1210 & 1 \\
\hline
\end{tabular}

By [13, Theorem 2(ii) and 2(iii)], each main class $[\mathcal{L}]$ splits into $\operatorname{Ty}(\mathcal{L})$ isomorphism classes of one-factorizations of $K_{n, n}$ (cf. 4 .1.1) and into $3 ! \cdot|\operatorname{Is}(\mathcal{L})| /|\operatorname{Par}(\mathcal{L})|$ isotopy classes.

Taking the sum over all main classes, we obtain Theorem1(ii) and (iii). Table13 gives the order of the autoparatopy group and the order of the autotopy group for each main class.

5.3. Quasigroups and loops. A permutation with $a_{i}$ cycles of length $i$ for $i \geq 1$ is said to have cycle structure $\left(a_{1}, a_{2}, \ldots\right)$. For an autotopism $\alpha \in \operatorname{Is}(\mathcal{L})$, define $f(\alpha)=\prod_{i} a_{i} ! i^{a_{i}}$ and $g(\alpha)=a_{1}$ if $\alpha$ is not the identity permutation and has the same cycle structure $\left(a_{1}, a_{2}, \ldots\right)$ in each of the point classes $R, C, S$; otherwise $f(\alpha)=g(\alpha)=0$. 
The number of isomorphism classes of quasigroups is, by [13, Theorem 4],

$$
\frac{|\mathscr{L}|}{n !}+\sum_{[\mathcal{L}]} \frac{3 !}{|\operatorname{Par}(\mathcal{L})|} \sum_{\alpha \in \operatorname{Is}(\mathcal{L})} f(\alpha)^{2} .
$$

The number of isomorphism classes of loops is, by [13, Theorem 5],

$$
\frac{|\mathscr{L}|}{n ! \cdot(n-1) !^{2}}+\sum_{[\mathcal{L}]} \frac{3 !}{|\operatorname{Par}(\mathcal{L})|} \sum_{\alpha \in \operatorname{Is}(\mathcal{L})} g(\alpha)^{2} .
$$

We obtain Theorem 1(iv) and (v) by (19), (20), (21), and summation over the main classes.

\section{ACKNOWLEDGMENTS}

The authors thank Ian Wanless and an anonymous referee for valuable comments and corrections. This research was supported in part by the Academy of Finland, Grants 107493, 110196, 117499, 130142, and 132122; as well as by the National Science Foundation, Grant Number 0633333.

\section{REFERENCES}

[1] C.J. Colbourn, J.H. Dinitz, and I.M. Wanless, Latin squares, in: Handbook of Combinatorial Designs, C.J. Colbourn and J.H. Dinitz, Eds., Second edition, Chapman \& Hall/CRC, Boca Raton (2007) pp. 135-152. MR2246267 (2007i:05001)

[2] J.H. Dinitz, D.K. Garnick, and B.D. McKay, There are 526,915,620 nonisomorphic onefactorizations of $K_{12}$, J. Combin. Des. 2 (1994) 273-285. MR.1276829 (95f:05089)

[3] The GAP Group, GAP - Groups, Algorithms, and Programming, Version 4.4.12; 2008. (http://www.gap-system.org)

[4] GNU Multiple Precision Arithmetic Library, Version 4.2.1. (http://gmplib.org/)

[5] P. Kaski, Isomorph-free exhaustive generation of designs with prescribed groups of automorphisms, SIAM J. Discrete Math. 19 (2005) 664-690. MR2191287 (2006h:05038)

[6] P. Kaski and P.R.J. Östergård, Classification Algorithms for Codes and Designs, Springer, Berlin, 2006. MR2192256 (2008a:05002)

[7] P. Kaski and P.R.J. Östergård, There are 1,132,835,421,602,062,347 nonisomorphic onefactorizations of $K_{14}$, J. Combin. Des. 17 (2009) 147-159. MR2489440

[8] P. Kaski and O. Pottonen, libexact User's Guide, Version 1.0, HIIT Technical Reports 2008-1, Helsinki Institute for Information Technology HIIT, 2008.

[9] G. Kolesova, C.W.H. Lam, and L. Thiel, On the number of $8 \times 8$ Latin squares, J. Combin. Theory Ser. A 54 (1990) 143-148. MR.1051788 (91d:05024)

[10] B.D. McKay, nauty User's Guide (Version 1.5), Technical Report TR-CS-90-02, Computer Science Department, Australian National University, Canberra, 1990.

[11] B.D. McKay, autoson - A Distributed Batch System for UNIX Workstation Networks (Version 1.3), Technical Report TR-CS-96-03, Computer Science Department, Australian National University, 1996.

[12] B.D. McKay, Isomorph-free exhaustive generation, J. Algorithms 26 (1998) 306-324. MR.1606516 (98k:68132)

[13] B.D. McKay, A. Meynert, and W. Myrvold, Small Latin squares, quasigroups, and loops, J. Combin. Des. 15 (2007) 98-119. MR2291523 (2007j:05030)

[14] B.D. McKay and I.M. Wanless, On the number of Latin squares, Ann. Comb. 9 (2005) 335344. MR2176596 (2006f:05027)

[15] H.W. Norton, The $7 \times 7$ squares, Ann. Eugenics 9 (1939) 269-307. MR0001220 (1:199a)

[16] J.J. Rotman, An Introduction to the Theory of Groups, Fourth edition, Springer, New York, 1995. MR1307623 (95m:20001)

[17] A. Sade, An omission in Norton's list of $7 \times 7$ squares, Ann. Math. Statistics 22 (1951) 306-307. MR0040254(12:665a) 
[18] D.S. Stones and I.M. Wanless, Divisors of the number of Latin rectangles, J. Combin. Theory Ser. A 117 (2010), 204-215. MR.2557890

[19] D.B. West, Introduction to Graph Theory, Second edition, Prentice-Hall, Upper Saddle River, 2001. MR1367739 (96i:05001)

Department of Mathematics, Colorado State University, 1874 Campus Delivery, Fort Collins, Colorado 80523-1874

E-mail address: hulpke@math.colostate.edu

Helsinki Institute for Information Technology Hitt, University of Helsinki, Department of Computer Science, P.O. Box 68, 00014 University of Helsinki, Finland

E-mail address: petteri.kaski@cs.helsinki.fi

Department of Communications and Networking, Aalto University, P.O. Box 13000, 00076 Aalto, Finland

E-mail address: patric.ostergard@tkk.fi 